\title{
Complete treatment of single-photon emission in planar channeling
}

\author{
Tobias N. Wistisen and Antonino Di Piazza \\ Max-Planck-Institut für Kernphysik, Saupfercheckweg 1 D-69117, Germany
}

(Received 5 April 2019; published 13 June 2019)

\begin{abstract}
Approximate solutions of the Dirac equation are found for ultrarelativistic particles moving in a periodic potential, which depends only on one coordinate, transverse to the largest component of the momentum of the incoming particle. As an example, we employ these solutions to calculate the radiation emission of positrons and electrons trapped in the planar potential found between the (110) planes in silicon. This allows us to compare with the semiclassical method of Baier, Katkov, and Strakhovenko, which includes the effect of spin and photon recoil but neglects the quantization of the transverse motion. For high-energy electrons, the high-energy part of the angularly integrated photon energy spectrum calculated with the found wave functions differs from the corresponding one calculated with the semiclassical method. However, for lower particle energies, it is found that the angularly integrated emission energy spectra obtained via the semiclassical method is in fairly good agreement with the full quantum calculation except that the positions of the harmonic peaks in photon energy and the photon emission angles are shifted.
\end{abstract}

DOI: $10.1103 /$ PhysRevD.99.116010

\section{INTRODUCTION}

Under certain circumstances, when a high-energy charged particle enters a crystalline medium, the particle dynamics is not dominated by the scattering on single atoms but rather by the coherent scattering on many atoms, resulting in a smooth, bound motion along crystal axes or planes [1]. This motion leads to radiation emission called channeling radiation. This has been studied both experimentally [2-13] and theoretically [5,14-17]. Channeling radiation from high-energy electrons/positrons represents one of the few experimental realizations of nonperturbative and nonlinear problems in quantum electrodynamics, where the field strength experienced by the particle in its rest frame approaches the Schwinger field $E_{\text {cr }}=1.3 \times$ $10^{16} \mathrm{~V} / \mathrm{cm}$, see Fig. 1 . The only other experiments where this has been realized were the SLAC laser experiment [18] and the recently reported experiments [19,20], although they had a smaller quantum nonlinearity parameter. The quantum nonlinearity parameter is the ratio of the field strength experienced by a charged particle in its rest frame and the Schwinger field strength. While the quantum treatment of radiation emission in a laser field can be fully treated using the Volkov state [21-27], no such full treatment has been presented for channeling radiation. The theory of channeling radiation so far has consisted of a

Published by the American Physical Society under the terms of the Creative Commons Attribution 4.0 International license. Further distribution of this work must maintain attribution to the author(s) and the published article's title, journal citation, and DOI. Funded by SCOAP . quantum approach involving wave functions at low particle energies, but which neglects spin, photon recoil, and nondipole transitions in the emission process because these effects are not important at low energies as compared to the effect of the quantization of the energy levels in the potential. Such an approach can be found, for example, in Refs. $[6,28,29]$. For higher particle energies, the theory relies on the semiclassical operator method by Baier et al. [30], which then includes the effects of spin and photon recoil but neglects the quantization of the transverse motion. The semiclassical method is relatively easy to implement numerically, and this explains why this method is often employed for numerical calculations in crystal channeling and crystalline undulators [31-33]. In many of the original theoretical works on channeling radiation, the connection between the Dirac equation and a
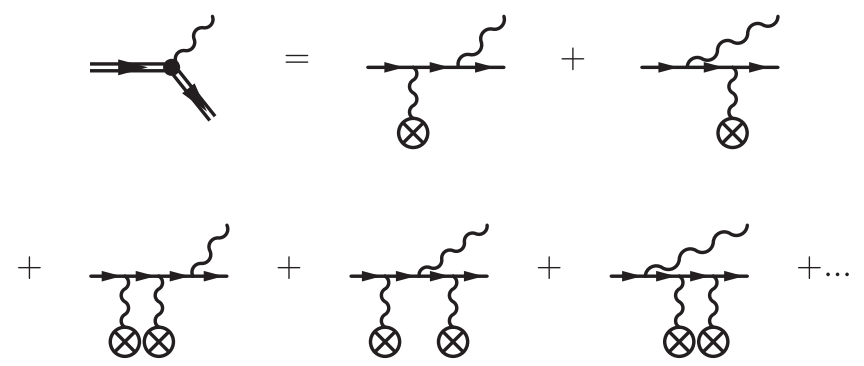

FIG. 1. The Feynman diagrams corresponding to the process under study. The double fermion lines correspond to the electron solutions of the Dirac equation in the background field of the inter planar crystal potential, which in the perturbative picture corresponds to including all orders of interactions with this background field. 
Schrödinger-like equation was seen, but then various approximations were employed, such as a simplified potential yielding analytical solutions, the dipole approximation in the calculation of the radiation, and/or the assumption that $\omega / \varepsilon$ is small, where $\omega$ is the energy of the emitted photon and $\varepsilon$ is the initial energy of the radiating particle; see Refs. [34-38]. See also Refs. [39-53] for recent advancements on the subject of crystal channeling.

In this paper, we present an investigation of planar channeling, based on wave functions that are approximate solutions of the Dirac equation in the realistic Doyle-Turner model of the periodic crystal potential and calculate the single-photon radiation emission without any of the mentioned approximations. Therefore, we now include all relevant quantum effects, which in some cases yield differences as compared to the semiclassical theory. In particular, the fact that the radiation emission stems from transitions between discrete bound states between the planes is properly taken into account. We investigate an example of $20 \mathrm{GeV}$ planar channeled electrons, as in this case one can have significant radiation emission from bound states with a low quantum number. In this case, the quantization of the motion is important, and at the same time, photon energies comparable to the electron energy are emitted, such that spin and photon recoil effects are also important. In addition, for very high particle energies, transitions from bound states with high to low quantum numbers become more likely. Therefore, the high-energy part of the spectrum is different from that obtained via the semiclassical model, as states with low quantum numbers are only approximately accounted for by the latter model. As an example, we will see this effect for planar channeled electrons with initial energy of $250 \mathrm{GeV}$ and $1 \mathrm{TeV}$.

We use units where $\hbar=c=1, \alpha=e^{2}$, with $e$ being the positron charge, and the Feynman slash notation such that $\not h=a_{\mu} \gamma^{\mu}$, where $\gamma^{\mu}$ are the Dirac gamma matrices and $a^{\mu}$ is an arbitrary 4-vector. We adopt the metric tensor $\eta^{\mu \nu}=$ $\operatorname{diag}(+1,-1,-1,-1)$. Below, when the term "particle" is employed, it will refer to either an electron or to a positron.

\section{WAVE FUNCTIONS}

In Ref. [54], we discussed the Dirac equation in a parabolic potential in the regime where $\xi \ll \gamma$, where $\xi$ is the typical transverse momentum (here, $p_{y}$ ) divided by the electron mass $m$ and $\gamma$ is the Lorentz gamma factor of the incoming particle. For channeling, we have that $\xi=\gamma \theta_{c}$, where $\theta_{c}=\sqrt{2 V_{0} / \varepsilon}$ is the critical angle for channeling [1], and so the requirement of validity reduces to $\theta_{c} \ll 1$ or $\varepsilon \gg V_{0}$. For silicon, $V_{0}=22.7 \mathrm{eV}$, and we are interested in ultrarelativistic particles with energy on the scale of $\mathrm{GeV}$, such that this approximation is safely applicable. Note that at $\xi \gg 1$ the overall emission angle (approximately $2 \xi / \gamma$ ) is much larger than the instantaneous one (approximately $1 / \gamma$ ), and this corresponds to the regime where the local constant field approximation (LCFA), in general, becomes applicable [55]. When $\xi$ is small, however, the dipole approximation may be used [55]. Another important parameter is the so-called quantum nonlinearity parameter already mentioned in the Introduction and defined by

$$
\chi=\frac{e \sqrt{\left|\left(F^{\mu \nu} p_{\nu}\right)^{2}\right|}}{m^{3}} .
$$

When $\chi$ becomes on the order of unity, the effects of particle spin and photon recoil become important in the radiation emission process. The quantum description of radiation emission using wave functions as seen in Refs. $[6,28,29]$ is valid when $\chi \ll 1$, because spin effects and recoil are neglected, and when $\xi \ll 1$, because the magnetic field in the particle's rest frame is neglected and only the dipole matrix element is calculated. In the present paper, we treat the problem from the laboratory frame, and the field can therefore be described solely by an electrostatic potential $\varphi(y)$. We then assume that the largest component of the particle momentum is along the $x$ direction. In Ref. [54] (and see Appendix A), we found that the positive-energy solutions of the Dirac equation, to leading order in $\xi / \gamma$, can be written as (we set the quantization volume $V=1$ )

$$
\psi(x)=\frac{1}{\sqrt{2 \varepsilon}} e^{i\left(p_{x} x+p_{z} z-\varepsilon t\right)} U(y),
$$

and $U(y)$ is given by

$$
U(y)=\sqrt{\varepsilon+m}\left(\begin{array}{c}
\boldsymbol{s} \\
\frac{\sigma \cdot \tilde{p}}{\varepsilon+m} \boldsymbol{s}
\end{array}\right) I(y),
$$

where $\tilde{\boldsymbol{p}}=\left(p_{x}-e \varphi(y),-i \frac{d}{d y}, p_{z}\right), \boldsymbol{s}$ is a two-component vector describing the spin, which we can choose as either $\left(\begin{array}{ll}1 & 0\end{array}\right)^{T}$ or $\left(\begin{array}{ll}0 & 1\end{array}\right)^{T}$, corresponding to spin-up and spindown, respectively. Note that in Eq. (3) we approximated

$$
\frac{\boldsymbol{\sigma} \cdot \boldsymbol{p}}{\varepsilon+m+e \varphi(y)} \simeq \frac{\boldsymbol{\sigma} \cdot \tilde{\boldsymbol{p}}}{\varepsilon+m},
$$

where $\boldsymbol{p}=\left(p_{x},-i \frac{d}{d y}, p_{z}\right)$; see also Appendix A. As already mentioned, we have taken $p_{x}$ to be the longitudinal direction, that is, in the initial state $p_{z}=0, p_{x}>0$ and $p_{x} \simeq \varepsilon \gg m \xi$, where $\varepsilon=m \gamma$ is the initial (constant) energy of the particle. The function $I(y)$ is the solution of the equation

$$
\left[-\frac{1}{2 \varepsilon} \frac{d^{2}}{d y^{2}}-e \varphi(y)\right] I(y)=\frac{\varepsilon^{2}-p_{x}^{2}-p_{z}^{2}-m^{2}}{2 \varepsilon} I(y) .
$$

For $\varphi(y)$, we will use the Doyle-Turner model [55-58], chosen as symmetric around $y=0$, which is then given by 
TABLE I. The parameters for the Doyle-Turner potential of silicon.

\begin{tabular}{lcc}
\hline \hline $\mathrm{i}$ & $a_{i}(\AA)$ & $B_{i}\left(\AA^{2}\right)$ \\
\hline 1 & 2.129 & 1.463 \\
2 & 2.533 & $4.173 \times 10^{-1}$ \\
3 & $8.349 \times 10^{-1}$ & $7.294 \times 10^{-2}$ \\
4 & $3.216 \times 10^{-1}$ & $9.777 \times 10^{-3}$ \\
\hline \hline
\end{tabular}

$$
\varphi(y)=\sum_{l=-\infty}^{\infty} \varphi_{1}\left(y-l d_{p}\right)
$$

where $d_{p}=1.92 \AA$ is the interplanar distance for the (110) planes in silicon,

$\varphi_{1}(y)=2 \sqrt{\pi} e a_{0} N d_{p} \sum_{i=1}^{4} \frac{a_{i}}{\sqrt{B_{i}+\rho^{2}}} \exp \left(-\frac{y^{2}}{B_{i}+\rho^{2}}\right)$,

$a_{0}$ is the Bohr radius, $N$ is the atomic number density, $\rho=0.106 \AA$ is the thermal vibrational amplitude for silicon, and the parameters $a_{i}$ and $B_{i}$ are given in Table I.

Clearly, Eq. (5) corresponds to an eigenvalue problem in the form we are accustomed to from atomic physics. The potential $\varphi(y)$ is periodic with the period $d_{p}$. Because of this periodicity, the solution can be written as a Bloch wave such that

$$
I(y)=e^{i k_{B} y} u_{k_{B}}(y)
$$

and where $u_{k_{B}}(y)$ is also periodic with period $d_{p}$. The quantity $k_{B}$ is the Bloch momentum, which can be taken to be in the interval $0 \leq k_{B}<k_{0}, k_{0}=\frac{2 \pi}{d_{p}}$. It then follows from Bloch's theorem that these solutions form an orthogonal and complete set of solutions of Eq. (5) [59] (see Appendix B for a proof that the resulting solutions of the Dirac equation are also orthonormal within our level of approximation). Now, we are interested in the solution $u_{k_{B}}(y)$, which is the nontrivial part of the wave function. Inserting $I(y)$ of Eq. (8) into Eq. (5) gives us the equation governing $u_{k_{B}}(y)$ :

$$
\begin{gathered}
{\left[-\frac{1}{2 \varepsilon}\left(\frac{d^{2}}{d y^{2}}+2 i k_{B} \frac{d}{d y}-k_{B}^{2}\right)+q \varphi(y)\right] u_{k_{B}}(y)} \\
=\frac{\varepsilon^{2}-p_{x}^{2}-p_{z}^{2}-m^{2}}{2 \varepsilon} u_{k_{B}}(y) .
\end{gathered}
$$

The periodicity of $u_{k_{B}}(y)$ implies that it can be written as the Fourier series

$$
u_{k_{B}}(y)=\sum_{j} c_{j} e^{i j k_{0} y} .
$$

To write any periodic function as a Fourier series, the sum includes infinitely many terms. However, for the numerical implementation, we are restricted to reducing the series to a finite sum. When the number of basis vectors is increased, it is found that the lowest lying states do converge, and therefore one only needs enough basis elements, that the sum describing the states of interest has converged to a fixed degree of accuracy. To ensure normalization, we should have $\sum_{j}\left|c_{j}\right|^{2}=1$ (see Appendix B). It is now clear that this is an eigenvalue problem where the quantized eigenvalue is

$$
E_{n}=\frac{\varepsilon^{2}-p_{x}^{2}-p_{z}^{2}-m^{2}}{2 \varepsilon},
$$

where $n$ is the quantum number corresponding to the value of this energy in ascending order and where $n=0$ corresponds the ground state. This equation leads to a quantization of, e.g., $p_{x}$. That is, if $\varepsilon$ is fixed by the incoming energy of the particle, a larger quantum number $n$ corresponds to a smaller value of $p_{x}$ in order to accommodate the larger transverse momentum in the $y$ direction. From this relation, it is also clear that the quantity $2 \varepsilon E_{n}$ is related to the square of the momentum in the $y$ direction, $p_{y}^{2}$. The coefficients $c_{j}$ are found by solving the matrix eigenvalue problem obtained by inserting Eq. (10) in Eq. (9), by multiplying by $e^{-i l k_{0} y} / d_{p}$, and by integrating over $y$ from $-d_{p} / 2$ to $d_{p} / 2$ to exploit orthogonality,

$$
\begin{aligned}
& \sum_{j} \frac{1}{2 \varepsilon}\left(j k_{0}+k_{B}\right)^{2} \delta_{j, l} c_{j} \\
& \quad+\sum_{j} c_{j} \frac{1}{d_{p}} \int q \varphi(y) e^{i(j-l) k_{0} y} d y \\
& =\sum_{j} \frac{\varepsilon^{2}-p_{x}^{2}-p_{z}^{2}-m^{2}}{2 \varepsilon} \delta_{j, l} c_{j},
\end{aligned}
$$

where $q=e$ for a positron and $q=-e$ for an electron. With these results taken into consideration, we now see that we can write the function $U(y)$ in terms of the coefficients $c_{j}$ such that

$$
U(y)=\sum_{j} c_{j} \boldsymbol{S}_{j} e^{i\left(j k_{0}+k_{B}\right) y},
$$

where

$$
\boldsymbol{S}_{j}=\sqrt{\varepsilon+m}\left(\begin{array}{c}
\boldsymbol{s} \\
\frac{\boldsymbol{\sigma} \cdot \boldsymbol{p}_{j}}{\varepsilon+m} \boldsymbol{s}
\end{array}\right)
$$

and where

$$
\boldsymbol{p}_{j}=\left(p_{x}+E_{n}-\frac{\left(j k_{0}+k_{B}\right)^{2}}{2 \varepsilon}, j k_{0}+k_{B}, p_{z}\right) .
$$

In order to write the momentum in this form, we have replaced the term with the potential by exploiting Eq. (9). 
Before calculating the radiation emission probability, we will calculate the expectation value of the momentum in the $y$ direction, as this will provide insight as to how the momentum relates to the quantum number $k_{B}$. By inserting our wave function from Eq. (2) in

$$
\left\langle p_{y}\right\rangle=\int_{-\infty}^{\infty} \psi^{\dagger}(x)\left(-i \frac{d}{d y}\right) \psi(x) d^{3} x,
$$

we find that this becomes simply

$$
\left\langle p_{y}\right\rangle=\sum_{j}\left|c_{j}\right|^{2}\left(j k_{0}+k_{B}\right),
$$

to leading order in our approximation; see Appendix C.

\section{SINGLE PHOTON EMISSION}

We will now derive the single photon emission probability. The leading-order $S$-matrix element for the emission of a single photon by an electron moving inside the potential $\varphi(y)$ is given by

$$
S_{i \rightarrow f}^{(1)}=-i \sqrt{\frac{4 \pi}{2 \omega}} \int_{-\infty}^{\infty} d^{4} x \bar{\psi}_{f}(x) e \phi^{*} e^{i k x} \psi_{i}(x),
$$

and the emission probability is then

$$
d P_{i \rightarrow f}^{(1)}=\left|S_{i \rightarrow f}^{(1)}\right|^{2} \frac{d^{3} p_{f}}{(2 \pi)^{3}} \frac{d^{3} k}{(2 \pi)^{3}} .
$$

By inserting our wave functions and integrating over the coordinates, which provide energy-momentum conservation delta functions, we have that

$$
\begin{aligned}
S_{i \rightarrow f}^{(1)}= & -i \mathcal{M}_{i \rightarrow f}(2 \pi)^{4} \delta\left(\varepsilon_{f}+\omega-\varepsilon_{i}\right) \\
& \times \delta\left(p_{x, i}-k_{x}-p_{x, f}\right) \delta\left(p_{z, i}-k_{z}-p_{z, f}\right) \\
& \times \delta\left(k_{B, i}-k_{y}-k_{B, f}-n_{B} k_{0}\right),
\end{aligned}
$$

where $n_{B}$ is the integer such that $0 \leq k_{B, f}<k_{0}$, henceforth denoted as the first Brillouin zone (FBZ) (see Appendix D for details on this derivation). In the above expression of the $S$-matrix element, we defined the reduced matrix element,

$\mathcal{M}_{i \rightarrow f}=e \sqrt{\frac{4 \pi}{2 \omega}} \frac{1}{2 \sqrt{\varepsilon_{f} \varepsilon_{i}}} \sum_{j} c_{n_{B}+j, f}^{*} c_{j, i} \overline{\boldsymbol{S}}_{n_{B}+j, f} \phi k \boldsymbol{S}_{j, i}$,

where $S_{j, i}$ corresponds to the initial state and $c_{j, i}$ is the coefficient with index $j$ corresponding to the initial state $i$. It is therefore seen that one must solve the matrix problem of Eq. (12) for $\varepsilon_{f}=\varepsilon_{i}-\omega$ several times, as the final state depends on the energy of the emitted photon $\omega$ and the Bloch momentum $k_{B, f}$ of the final electron (see Appendix D for additional details, especially on why $\mathcal{M}_{i \rightarrow f}$ reduces to a single sum over $j$ ). We find that the rate corresponding to Eq. (19), when dividing by total interaction time $T_{\text {int }}$ arising from squaring the energy delta function, is given by

$$
d W_{i \rightarrow f}^{(1)}=\frac{1}{(2 \pi)^{2}}\left|\mathcal{M}_{i \rightarrow f}\right|^{2} \delta\left(\varepsilon_{f}+\omega-\varepsilon_{i}\right) d^{3} k .
$$

As is usually the case when dealing with calculations concerning ultrarelativistic particles, it is pertinent to consider cancellations between the large terms in the expression in the delta function, $\varepsilon_{f}+\omega-\varepsilon_{i}$ because the relevant transverse energies $E_{n}$, comparable to the potential depth, are much smaller than the whole energy of the particle (recall that the former are of the order of several electron-volts, whereas the latter is of the order of gigaelectron-volts). For this, it is useful to consider the quantity $\Delta$, which we define via the equation $\varepsilon=p_{x}+\Delta$. Inserting this into the equation for our eigenvalues, Eq. (11), we obtain that to leading order in $E_{n} / \varepsilon$,

$$
\Delta=E_{n}+\frac{p_{z}^{2}+m^{2}}{2 \varepsilon} \text {. }
$$

Defining $\quad \boldsymbol{k}=\omega(\cos \theta, \sin \theta \cos \varphi, \sin \theta \sin \varphi)$, we can rewrite the argument of the remaining energy delta function as (note that in the present problem it is convenient to use the $x$ axis as the polar axis)

$$
\begin{aligned}
f(\theta)= & \varepsilon_{f}+\omega-\varepsilon_{i} \\
= & E_{n_{f}}-E_{n_{i}}+\frac{p_{z, f}^{2}+m^{2}}{2 \varepsilon_{f}}-\frac{p_{z, i}^{2}+m^{2}}{2 \varepsilon_{i}} \\
& +p_{x, f}-p_{x, i}+\omega \\
\simeq & E_{n_{f}}-E_{n_{i}}+\frac{m^{2}}{2 \varepsilon_{f}}-\frac{m^{2}}{2 \varepsilon_{i}} \\
& +\frac{\omega \theta^{2}}{2}\left(1+\frac{\omega \sin ^{2} \varphi}{\varepsilon_{f}}\right) .
\end{aligned}
$$

We define $\theta_{0}$ as the positive solution of the equation $f\left(\theta_{0}\right)=0$. When considering bound states, the energies $E_{n}$ become nearly independent of $k_{B}$, as seen in Fig. 2. In this case, one can isolate the emission angle as

$$
\theta_{0}=\sqrt{\frac{2\left(E_{n_{i}}-E_{n_{f}}\right)+\frac{m^{2}}{\varepsilon_{i}}-\frac{m^{2}}{\varepsilon_{f}}}{\omega\left(1+\frac{\omega \sin ^{2} \varphi}{\varepsilon_{f}}\right)}},
$$

and the threshold for a given transition is given by the condition where the numerator vanishes, which gives us that

$$
\omega_{\mathrm{th}, \mathrm{q}}=\varepsilon_{i}\left(1-\frac{1}{1+\frac{2 \varepsilon_{i}\left(E_{n_{i}}-E_{n_{f}}\right)}{m^{2}}}\right) .
$$




\section{SEMICLASSICAL METHOD}

In order to compare with the classical theory, we will derive a formula for the emitted power in the case of a periodic transverse motion, and then in the end, this can be turned into a semiclassical formula by comparing with the expression of Baier et al. [see Eq. (28) below]. Classically, the emitted energy per unit frequency and solid angle can be written as $[60,61]$

$$
\frac{d^{2} I^{\mathrm{Cl}}}{d \omega d \Omega}=\frac{e^{2} \omega^{2}}{4 \pi^{2}}\left|\int_{-\infty}^{\infty}(\boldsymbol{n}-\boldsymbol{v}) e^{i k x} d t\right|^{2},
$$

where $k^{\mu}=(\omega, \boldsymbol{k})$ is the wave 4-vector of the emitted radiation, where $x^{\mu}=(t, \boldsymbol{r})$ is the 4-position of the particle, $\boldsymbol{v}$ its velocity, and $\boldsymbol{n}=\boldsymbol{k} / \omega$. In the semiclassical formalism, the same quantity is given by $[30,55,61]$

$$
\begin{aligned}
\frac{d^{2} I}{d \omega d \Omega}= & \frac{e^{2} \omega^{\prime 2}}{4 \pi^{2}}\left(\frac{\varepsilon^{2}+\varepsilon^{\prime 2}}{2 \varepsilon^{2}}\left|\int_{-\infty}^{\infty}(\boldsymbol{n}-\boldsymbol{v}) e^{i k^{\prime} x} d t\right|^{2},\right. \\
& \left.+\frac{\omega^{2} m^{2}}{2 \varepsilon^{4}}\left|\int_{-\infty}^{\infty} e^{i k^{\prime} x} d t\right|^{2}\right),
\end{aligned}
$$

where $\varepsilon^{\prime}=\varepsilon-\omega, \omega^{\prime}=\omega \varepsilon / \varepsilon^{\prime}$ and $k^{\prime}=k \varepsilon / \varepsilon^{\prime}$ (this result holds in the case when the sum over final particle spins and photon polarizations and the average over initial spins are taken). As was shown in Refs. [61,62], we need only to calculate the transverse components of the integrand for ultrarelativistic particles as the longitudinal component is suppressed by at least a factor of $1 / \gamma$ in comparison. Therefore, for the classical formula, we have to calculate

$$
\int_{-\infty}^{\infty} e^{i \omega(t-\boldsymbol{n} \cdot \boldsymbol{r})} d t=\int_{-\infty}^{\infty} e^{i \omega\left(t-\boldsymbol{n}_{\perp} \cdot \boldsymbol{r}_{\perp}-n_{x} x(t)\right)} d t
$$

Since most of the radiation is emitted in the forward direction for ultrarelativistic particles, we may perform the smallangle expansion, and so we write $n_{x}=1-\frac{\theta^{2}}{2}$ and $x(t)=$ $\left\langle v_{x}\right\rangle t+\delta x(t)$, where we exploit the fact that the motion is quasiperiodic. For this reason, the quantity $\delta x(t)$ is a periodic function. By using the fact that $v^{2}$ is approximately conserved, we have that $\left\langle v_{x}\right\rangle \simeq 1-\frac{1}{2 \gamma^{2}}-\frac{v_{\perp}^{2}}{2}$; see also Ref. [60]. Inserting this result in the above expression and canceling the large terms, we obtain

$$
\begin{aligned}
\int_{-\infty}^{\infty} e^{i \omega(t-\boldsymbol{n} \cdot \boldsymbol{r})} d t \\
\quad=\int_{-\infty}^{\infty} e^{i \omega\left[t\left(\frac{\theta^{2}}{2}+\frac{\left\langle\nu^{2}\right\rangle}{2}+\frac{1}{2 \gamma^{2}}\right)-\boldsymbol{n}_{\perp} \cdot \boldsymbol{r}_{\perp}-\delta x\right]} d t .
\end{aligned}
$$

Now, if $\left\langle\boldsymbol{v}_{\perp}\right\rangle$ is zero, i.e., we have chosen the coordinate system where this is the case, we can exploit that $\boldsymbol{r}_{\perp}$ and $\delta x$ are periodic and write $\int_{-\infty}^{\infty} f(t) d t=\sum_{l} \int_{l T}^{(l+1) T} f(t) d t$, where $T$ is the period of the motion, and change variable at which point the Dirichlet kernel appears, which can be replaced by a sum of delta functions. Thus, we obtain

$$
\begin{aligned}
& \int_{-\infty}^{\infty} e^{i \omega(t-n \cdot \boldsymbol{r})} d t \\
& =C(\omega, \theta) \sum_{l} \delta\left(\frac{\omega}{2 \gamma^{2} \omega_{0}}\left(1+\gamma^{2} \theta^{2}+\gamma^{2}\left\langle v_{\perp}^{2}\right\rangle\right)-l\right)
\end{aligned}
$$

where $\omega_{0}=\frac{2 \pi}{T}$ and where we introduced the function

$$
C(\omega, \theta)=\int_{0}^{T} e^{i \omega\left[t\left(\frac{\theta^{2}}{2}+\frac{\left\langle\nu^{2}\right\rangle}{2}+\frac{1}{2 \gamma^{2}}\right)-n_{\perp} \cdot r_{\perp}-\delta x\right]} d t
$$

Analogously, we introduce the quantity

$$
\boldsymbol{D}_{\perp}(\omega, \theta)=\int_{0}^{T} \boldsymbol{v}_{\perp} e^{i \omega\left[t\left(\frac{\theta^{2}}{2}+\frac{\left\langle v_{\perp}^{2}\right\rangle}{2}+\frac{1}{2 \gamma^{2}}\right)-\boldsymbol{n}_{\perp} \cdot \boldsymbol{r}_{\perp}-\delta x\right]} d t
$$

For the integral with the velocity, completely analogous steps can be taken, and therefore we have that

$$
\begin{aligned}
& \int_{-\infty}^{\infty} \boldsymbol{v}_{\perp} e^{i \omega(t-n \cdot \boldsymbol{r})} d t \\
& =\boldsymbol{D}_{\perp}(\omega, \theta) \sum_{l} \delta\left(\frac{\omega}{2 \gamma^{2} \omega_{0}}\left(1+\gamma^{2} \theta^{2}+\gamma^{2}\left\langle\boldsymbol{v}_{\perp}^{2}\right\rangle\right)-l\right) .
\end{aligned}
$$

By inserting these quantities into the classical formula and using that the delta function squared gives us the delta function again with a factor of $\Delta \varphi / 2 \pi=T_{\text {int }} \omega_{0} / 2 \pi$. Therefore, we obtain that the emitted energy per unit time $T_{\text {int }}$ is given by

$$
\begin{aligned}
\omega d W^{\mathrm{Cl}}= & \frac{e^{2} \omega}{4 \pi^{2}} \frac{\omega_{0}^{2}}{2 \pi} \\
& \times \sum_{l}\left(\left|C\left(\omega, \theta_{l, \mathrm{Cl}}\right) \boldsymbol{n}_{\perp}-\boldsymbol{D}_{\perp}\left(\omega, \theta_{l, \mathrm{Cl}}\right)\right|^{2}\right) d \omega d \varphi,
\end{aligned}
$$

where we used the delta function to integrate over the angle $\theta$ such that

$$
\theta_{l, \mathrm{Cl}}=\frac{1}{\gamma} \sqrt{\frac{2 \gamma^{2} \omega_{0} l}{\omega}-\left(1+\gamma^{2}\left\langle\boldsymbol{v}_{\perp}^{2}\right\rangle\right)} .
$$

Note that for the sake of convenience we have introduced here a "classical" emission probability, even though such a quantity has a meaning only within the quantum theory. By performing the appropriate substitutions of $\omega \rightarrow \omega^{\prime}$ and by putting in the front factors as in Eq. (28), we can obtain the semiclassical version of the result in the form 


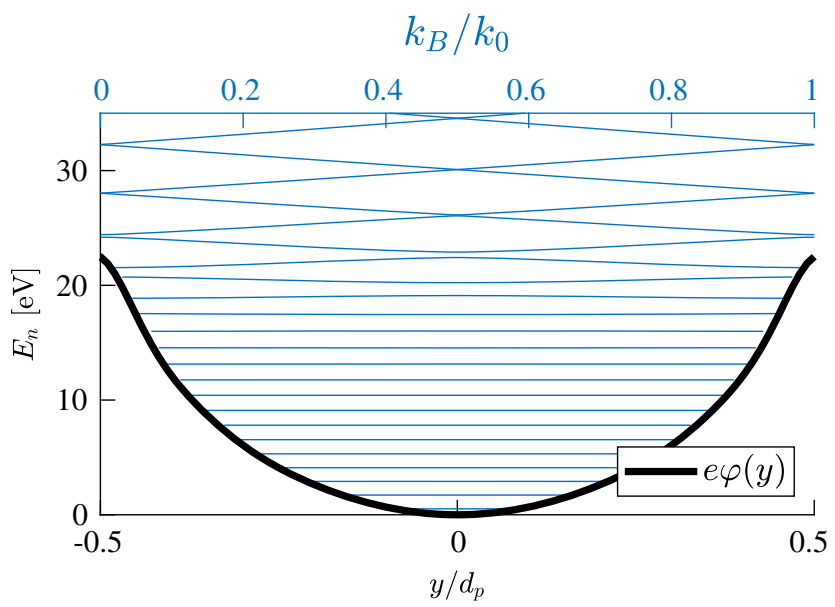

FIG. 2. The potential $\varphi(y)$ along with the positron energy bands in the first Brillouin zone for the energy $\varepsilon=100 \mathrm{MeV}$. This energy was chosen to have a reasonable number of levels so that the plot is not cluttered.

$$
\begin{aligned}
\omega d W= & \frac{e^{2} \omega^{\prime}}{4 \pi^{2}} \frac{\omega_{0}^{2}}{2 \pi} \sum_{l}\left(\frac{\varepsilon^{2}+\varepsilon^{\prime 2}}{2 \varepsilon^{2}}\left|C\left(\omega^{\prime}, \theta_{l}\right) \boldsymbol{n}_{\perp}-\boldsymbol{D}_{\perp}\left(\omega^{\prime}, \theta_{l}\right)\right|^{2}\right. \\
& \left.+\frac{\omega^{2} m^{2}}{2 \varepsilon^{4}}\left|C\left(\omega^{\prime}, \theta_{l}\right)\right|^{2}\right) d \omega d \varphi
\end{aligned}
$$

where

$$
\theta_{l}=\frac{1}{\gamma} \sqrt{\frac{2 \gamma^{2} \omega_{0} l}{\omega^{\prime}}-\left(1+\gamma^{2}\left\langle v_{\perp}^{2}\right\rangle\right)}
$$

The threshold is therefore found to be at

$$
\omega_{\mathrm{th}, \mathrm{B}}=\varepsilon \frac{2 \gamma \omega_{0} l}{m\left(1+\gamma^{2}\left\langle v_{\perp}^{2}\right\rangle\right)+2 \gamma \omega_{0} l} .
$$

\section{A. Local constant field approximation}

We will compare our results with the often used local constant field approximation. The emission rate in a constant field characterized by $\chi$ is given by [55]

$$
\begin{aligned}
\frac{d W}{d u}= & \frac{\alpha}{\pi \sqrt{3}} \frac{m^{2}}{\varepsilon} \frac{1}{(1+u)^{2}}\left\{\frac{1+(1+u)^{2}}{1+u} K_{2 / 3}\left(\frac{2 u}{3 \chi}\right)\right. \\
& \left.-\int_{2 u / 3 \chi}^{\infty} K_{1 / 3}(z) d z\right\},
\end{aligned}
$$

where $u=\omega /(\varepsilon-\omega)$ and $K_{\nu}$ is the modified Bessel function of the second kind of order $\nu$. The LCFA then amounts to calculating the Lorentz force trajectory through the external field, and then one can evaluate $\chi(t)$, and the emission rate is then averaged over times using the above formula. In the current case, one can average over the period $T$. The formula of Eq. (40) can be derived directly

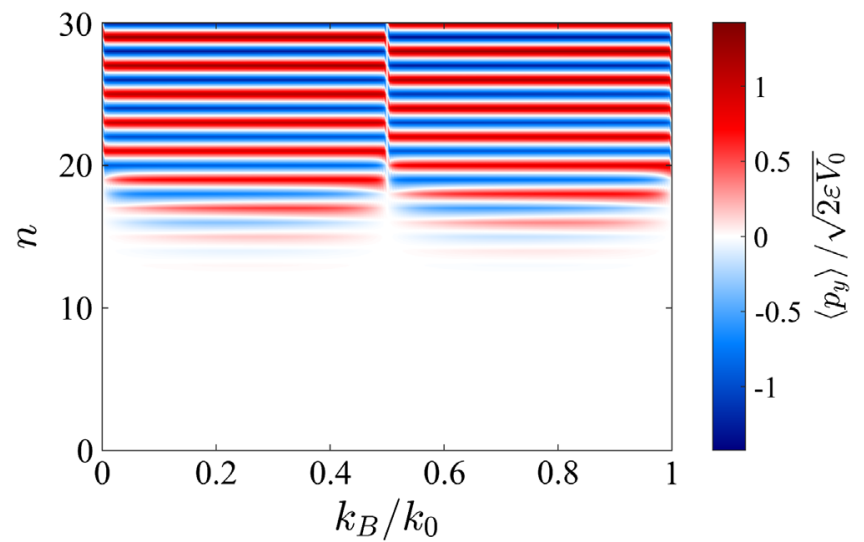

FIG. 3. A plot of the expectation value of $p_{y}$ as function of the quantum numbers $k_{B}$ and $n$ for a $100 \mathrm{MeV}$ positron, corresponding to Fig. 1.

from Eq. (28) by writing it as a double integration over two times $t_{1}=t-\tau / 2$ and $t_{2}=t+\tau / 2$ and performing the Taylor expansion of the trajectory $\boldsymbol{r}(t \pm \tau / 2)$ in $\tau$ in terms of the velocity and acceleration at time $t$ and keeping only terms up to the acceleration as was done in Ref. [55]; therefore, the semiclassical spectra will go towards the LCFA spectra when it is adequate to keep only these terms, and this is the case when the field varies slowly over the formation length $l_{f}=2 \gamma^{2}(1-\omega / \varepsilon) / \omega$ [55], and therefore for large values of $\omega$, it is expected that the SC spectra agree with the LCFA.

\section{DISCUSSION OF RESULTS}

In Fig. 2, we show the energy bands for a positron with $100 \mathrm{MeV}$ in the Doyle-Turner potential describing the (110) planes of silicon. It is noted that the energy $E_{n}$ of bound states with small values of $n$ is almost independent of $k_{B}$. In Fig. 3, we show a plot of the expectation value of the transverse momentum $p_{y}$, and we see that the bound states, with $E_{n}<V_{0}$, have $\left\langle p_{y}\right\rangle \simeq 0$, and for the states above the barrier, the quantity $\left\langle p_{y}\right\rangle$ steadily increases with $E_{n}$. Also, it depends on $k_{B}$ in such a way that half of the FBZ describes particles going to the left and the other half describes particles going to the right. In Fig. 4, we show the total power of a $250 \mathrm{GeV}$ electron/positron, calculated with the LCFA, depending on the initial position $y_{0}$, when assuming the beam angular divergence is negligible, i.e., much smaller than the critical angle $\theta_{c}$. What can be seen from this figure is that in both cases of the electron and positron, particles which start out close to the plane (here, $y_{0}=0$ ) have a larger radiation power. However, for positrons, as they are repelled from the planes, this means a large oscillation amplitude and therefore large $n$ quantum number, while for electrons, which are attracted to the planes, starting close to the plane means a small oscillation amplitude, and therefore small $n$ quantum numbers radiate 


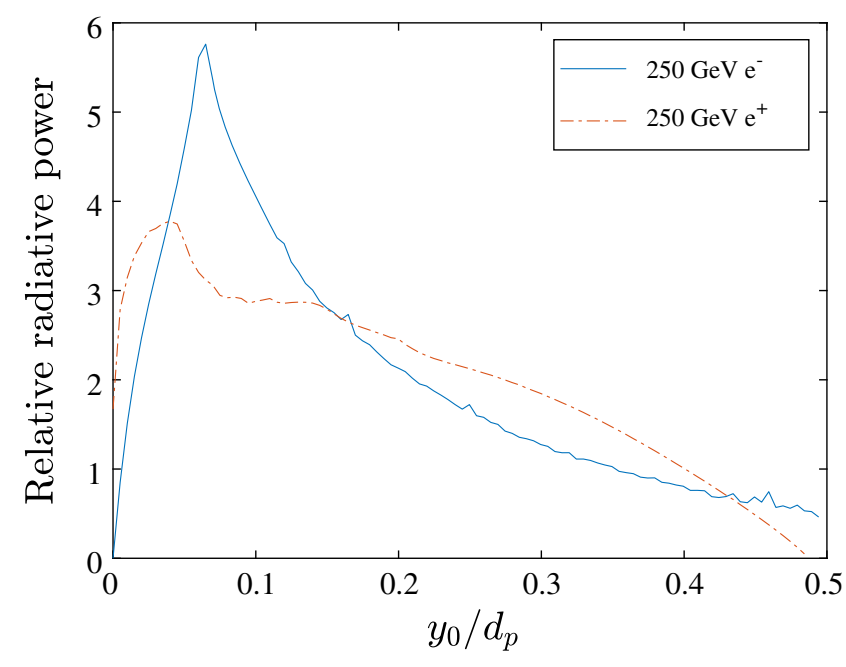

FIG. 4. The total power, relative to the average, of a $250 \mathrm{GeV}$ electron/positron incident at the position $y_{0}$ with zero angle.

more for electrons. This, combined with the fact that electrons will generally have an emission spectrum distributed around larger photon energies, implies that all the quantum effects mentioned in the Introduction may become important. In Fig. 5, we show the calculation of the photon emission spectra for a $20 \mathrm{GeV}$ electron in the state with $n=20$. This value of $n$ was picked as states around this value of $n$ have the largest rate of emission. In the particular case seen in Fig. 5, we can see the influence of the quantum effects in the following way. The fact that the classical calculation differs from the semiclassical method of Baier et al. means that the effects of spin and of photon recoil are present, i.e., that $\chi$ is large enough that these effects are sizable. At the same time, we see that the calculations presented here differ from the semiclassical of Baier et al. because the quantum number, $n=20$, of this state is not large enough that the quantization of the motion can be completely neglected. In addition, we have that $\xi$ is on the order of unity, and therefore one can apply neither the constant field approximation nor the dipole approximation. We see, however, in Fig. 5 that the overall level of the semiclassical spectrum falls together with the quantum results obtained here, while the most noticeable difference is in the position of the thresholds, which should also be clear from Eqs. (26) and (39); see Refs. [54,63], where this is also found for different field configurations. Here, it is seen that the threshold depends critically on the difference in energy between the quantized levels in the transverse potential, information which is not contained in the semiclassical method. In an experiment, one would, however, only obtain an average over the spectra corresponding to different states, and therefore these details would likely be washed out. Definitive distinction could, however, be observed if the emitted photon energy $\omega$ could be measured along with the emission angles $\theta$ and $\varphi$. This can be seen from Eqs. (25) and (38), which show that also the emission

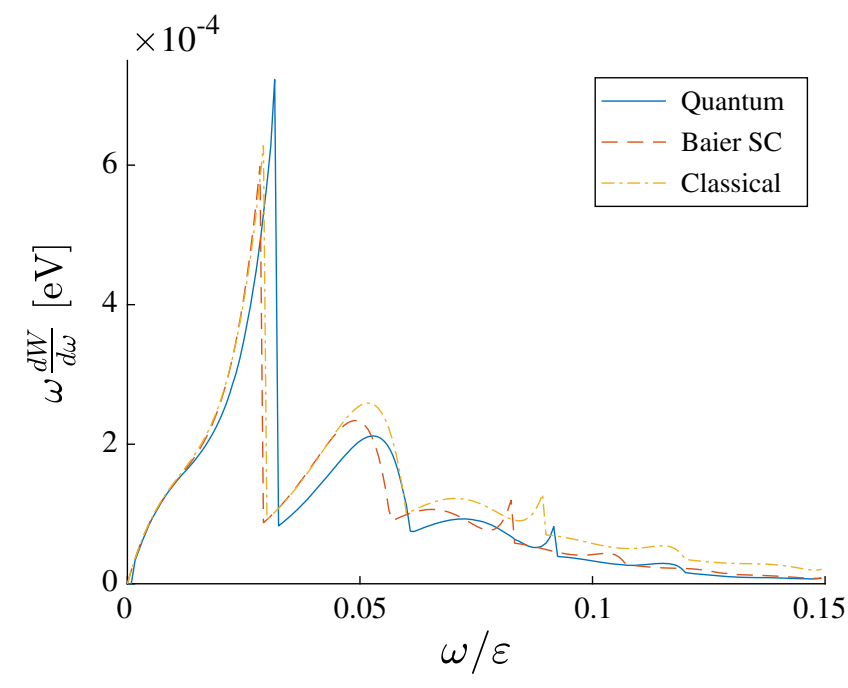

FIG. 5. The differential power (probability per unit time multiplied by the photon energy) for the case of a $20 \mathrm{GeV}$ electron in the state $n=20$ between the (110) planes of silicon. The label "quantum" is the calculation carried out by employing the wave functions found in this paper, "Baier SC" is the semiclassical method of Baier et al. for a particle with the same transverse mechanical energy, and "classical" is using the classical formula for radiation emission.

angle depends on the level spacing. In addition, a qualitative difference appears: the semiclassical treatment predicts that $\theta$ depends only on $\omega$, while in the full calculation presented here, there is also a dependence on $\varphi$ when $\omega \sim \varepsilon-\omega$, i.e., when $\chi$ is no longer small. In Fig. 6, we show that, while the same differences of the position of the thresholds can be seen here, the effect is very small for positrons with larger values of $n$ typically, as explained. However, if we picked a positron in a lower lying state, differences in the spectrum comparable to those seen in Fig. 5 would be seen, but such low lying states have a low total radiated power for positrons and therefore do not contribute much to the spectrum when averaged over initial conditions. In Fig. 7, we show the calculation for a $250 \mathrm{GeV}$ electron in the $n=45$ state, which corresponds to the particles with the largest power, i.e., the peak in Fig. 4 for electrons. We compare to the semiclassical, classical, and LCFA models. As expected, the LCFA approximation agrees with the semiclassical one for large photon energies, where the formation length is short. It is seen that the classical calculation fails for large photon energies, as expected. For the very high-energy part of the spectrum, it is also observed that the full quantum calculation is different from the semiclassical result. From Eq. (26), it can be seen that larger values of the difference $E_{n_{i}}-E_{n_{f}}$ imply a larger value of the emitted energy threshold. Therefore, the high-energy part of the photon spectrum arises from transitions where the electron goes from the initial state with $n=45$ to a state with a low value of the quantum number $n$. Now, in the semiclassical method, 


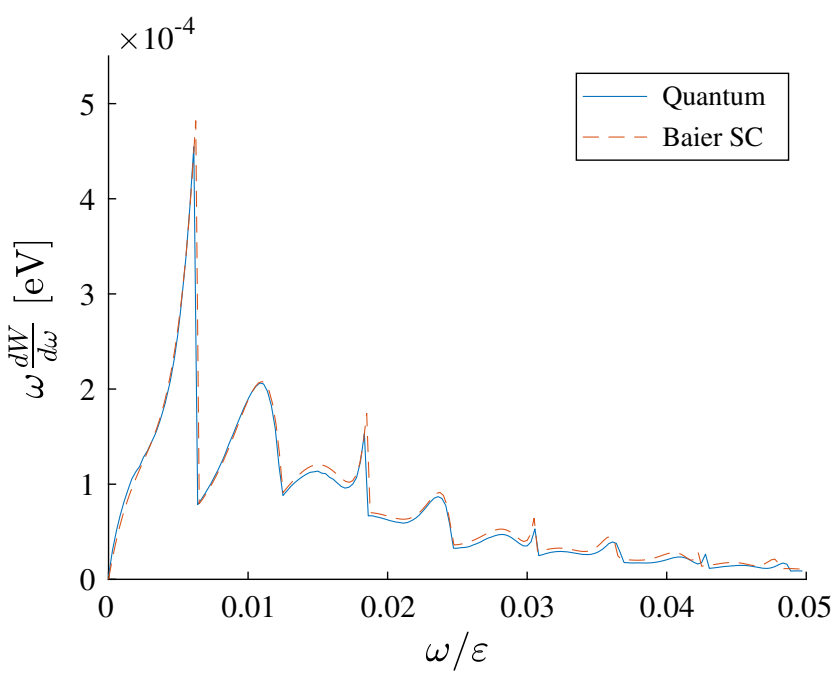

FIG. 6. The differential power (probability per unit time multiplied by the photon energy) for the case of a $10 \mathrm{GeV}$ positron in the state $n=150$ between the (110) planes of silicon. The label "quantum" is the calculation carried out by employing the wave functions found in this paper, and "Baier SC" is the semiclassical method of Baier et al. for a particle with the same transverse mechanical energy.

the wave functions of both initial and final states are approximated in a way which is only valid when the corresponding discrete quantum numbers are large. Since this is not the case for the final state, the semiclassical result differs from the full quantum one. In Fig. 8, the same effect is seen for the case of a $1 \mathrm{TeV}$ electron in the $n=120$ state initially. In this case, the effect is more pronounced and far above the level of the Bethe-Heitler bremsstrahlung, which is approximately $\omega d W_{\mathrm{BH}} / d \omega \simeq 2.8 \times 10^{-6} \mathrm{eV}$; see the section on "Passage of Particles Through Matter" in Ref. [64]. Therefore, if one carries out a precise measurement of the high-energy part of the spectrum, the discrepancy between the two models could be experimentally tested. In Fig. 9, we show the spectra for a $1 \mathrm{TeV}$ positron with a large $n$ quantum number, and in this case, the semiclassical method works well.

\section{CONCLUSION}

In conclusion, we have shown how to find approximate solutions of the Dirac equation for describing the motion of relativistic electrons and positrons in a periodic potential which depends on one transverse coordinate, as compared to the direction of the largest momentum (here indicated as the $x$ direction). We have shown how to calculate the emission rate of a single photon from transitions between the corresponding quantum states exactly, that is, without the use of either the dipole approximation or the local constant field approximation. Therefore, we have been able to calculate single-photon planar channeling radiation, with all relevant quantum effects included: the effects of

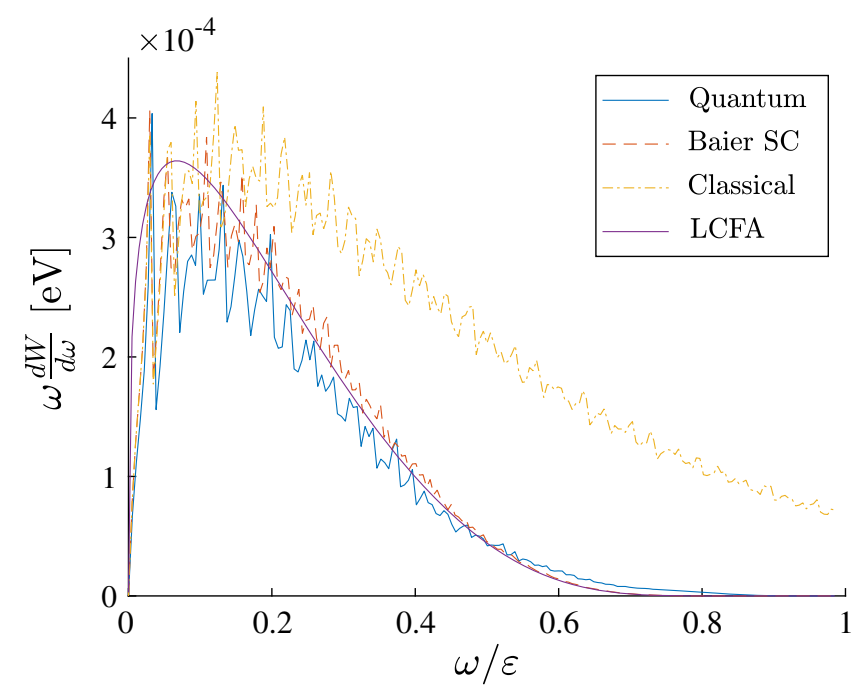

FIG. 7. The differential power (probability per unit time multiplied by the photon energy) for the case of a $250 \mathrm{GeV}$ electron in the state $n=45$ between the (110) planes of silicon. The label "quantum" is the calculation carried out by employing the wave functions found in this paper, "Baier SC" is the semiclassical method of Baier et al. for a particle with the same transverse mechanical energy, "classical" is using the classical formula for radiation emission, and LCFA is the local constant field approximation.

electron/positron spin and photon recoil during the emission, which the semiclassical method also incorporates, but also the effects of the quantization of the transverse motion. For planar channeling, and in particular for positrons, we saw that the semiclassical approximation of Baier et al. (beyond the local constant field approximation) is accurate in describing the energy distribution of the emitted photon, when integrated over angles. For electrons, differences are more noticeable. For low electron energies, a clear experimental measurement of such differences would require angular resolution of the emitted photons along with their energy. However, for higher-energy electrons, a difference could potentially be detected experimentally, even in the angularly integrated emission spectrum for emitted photons with high energy.

\section{ACKNOWLEDGMENTS}

T. W. is supported by the Alexander von HumboldtStiftung apart from the initial part of the project where funding was provided by the VILLUM FONDEN (research Grant No. VKR023371). In addition, the author acknowledges the support of NVIDIA Corporation with the donation of the Titan V GPU used for this research.

\section{APPENDIX A}

The general (un-normalized) solution to the Dirac equation with potential energy $V(\boldsymbol{r})=-e \varphi(\boldsymbol{r})$ can be written as 


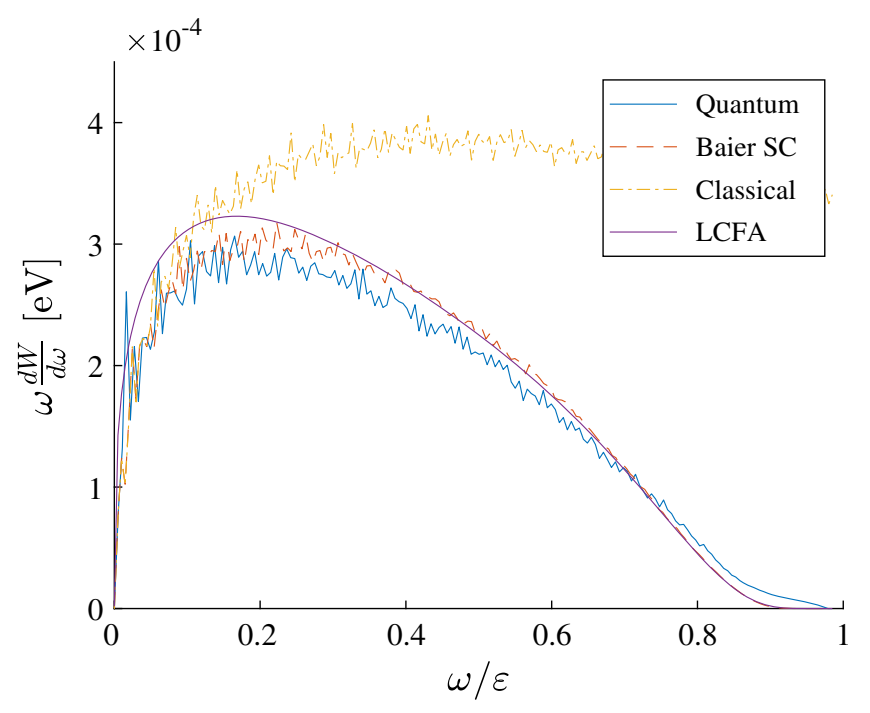

FIG. 8. The differential power (probability per unit time multiplied by the photon energy) for the case of a $1 \mathrm{TeV}$ electron in the state $n=120$ between the (110) planes of silicon. The labels have the same meaning as in Fig. 7.

$$
\psi(\boldsymbol{r}, t)=e^{-i \varepsilon t}\left(\begin{array}{l}
\phi(\boldsymbol{r}) \\
\chi(\boldsymbol{r})
\end{array}\right) .
$$

The Dirac equation then becomes

$$
\begin{aligned}
& (\varepsilon+e \varphi-m) \phi(\boldsymbol{r})=\boldsymbol{\sigma} \cdot \hat{\boldsymbol{p}} \chi(\boldsymbol{r}), \\
& (\varepsilon+e \varphi+m) \chi(\boldsymbol{r})=\boldsymbol{\sigma} \cdot \hat{\boldsymbol{p}} \phi(\boldsymbol{r}) .
\end{aligned}
$$

If we insert $\chi(\boldsymbol{r})$ from Eq. (A3) in Eq. (A2), we can obtain an equation for $\phi(\boldsymbol{r})$ :

$$
\begin{aligned}
& (\varepsilon+e \varphi-m) \phi(\boldsymbol{r}) \\
& =\boldsymbol{\sigma} \cdot \hat{\boldsymbol{p}} \frac{1}{(\varepsilon+e \varphi+m)} \boldsymbol{\sigma} \cdot \hat{\boldsymbol{p}} \phi(\boldsymbol{r}) \\
& =\frac{1}{(\varepsilon+e \varphi+m)}[\boldsymbol{\sigma} \cdot \hat{\boldsymbol{p}}]^{2} \phi(\boldsymbol{r})-i \frac{e \boldsymbol{\sigma} \cdot \boldsymbol{E}}{(\varepsilon+e \varphi+m)^{2}} \boldsymbol{\sigma} \cdot \hat{\boldsymbol{p}} \phi(\boldsymbol{r}) \\
& =\frac{1}{(\varepsilon+e \varphi+m)} \hat{\boldsymbol{p}}^{2} \phi(\boldsymbol{r})-i \frac{e \boldsymbol{\sigma} \cdot \boldsymbol{E}}{(\varepsilon+e \varphi+m)^{2}} \boldsymbol{\sigma} \cdot \hat{\boldsymbol{p}} \phi(\boldsymbol{r}) .
\end{aligned}
$$

Now, let us multiply with $(\varepsilon+e \varphi+m)$; then,

$$
\begin{aligned}
& \hat{\boldsymbol{p}}^{2} \phi(\boldsymbol{r})-i \frac{e \boldsymbol{\sigma} \cdot \boldsymbol{E}}{\varepsilon+e \varphi+m} \boldsymbol{\sigma} \cdot \hat{\boldsymbol{p}} \phi(\boldsymbol{r}) \\
& =(\varepsilon+e \varphi+m)(\varepsilon+e \varphi-m) \phi(\boldsymbol{r}) \\
& =\left[(\varepsilon+e \varphi)^{2}-m^{2}\right] \phi(\boldsymbol{r}) \\
& =\left[\varepsilon^{2}+2 \varepsilon e \varphi+e^{2} \varphi^{2}-m^{2}\right] \phi(\boldsymbol{r}) .
\end{aligned}
$$

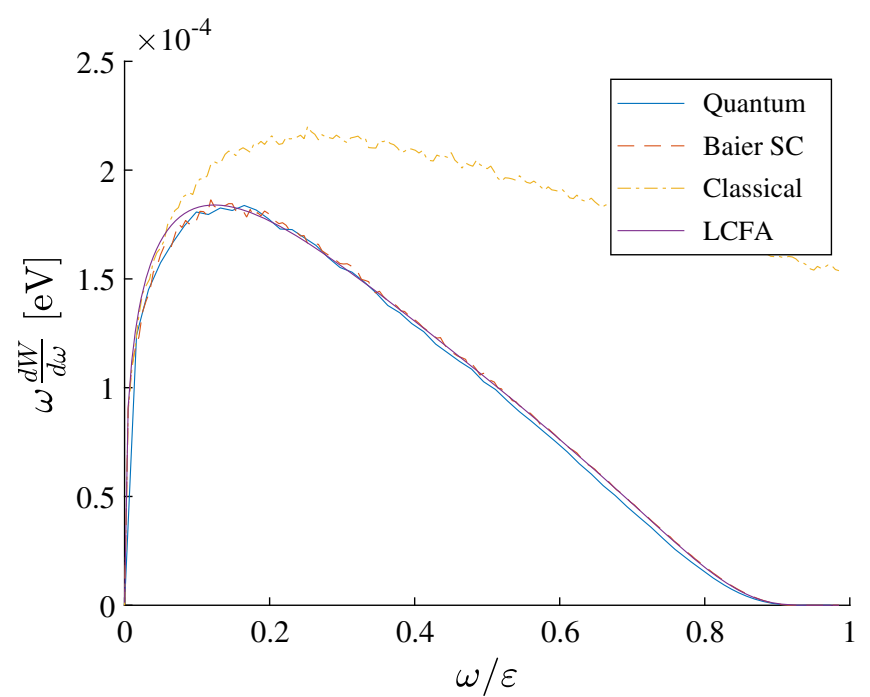

FIG. 9. The differential power (probability per unit time multiplied by the photon energy) for the case of a $1 \mathrm{TeV}$ positron in the state $n=1512$ between the (110) planes of silicon. The labels have the same meaning as in Fig. 7.

Now, if we neglect the spin-field interaction terms and the field squared term $e^{2} \varphi^{2}$, this becomes

$$
\left[\frac{1}{2 \varepsilon} \hat{\boldsymbol{p}}^{2}-e \varphi\right] \phi(\boldsymbol{r})=\frac{\varepsilon^{2}-m^{2}}{2 \varepsilon} \phi(\boldsymbol{r})
$$

The electron solution is then

$$
\psi(\boldsymbol{r}, t)=e^{-i \varepsilon t}\left(\begin{array}{c}
\phi(\boldsymbol{r}) \\
\frac{\boldsymbol{\sigma} \cdot \hat{\boldsymbol{p}}}{\varepsilon-V(\boldsymbol{r})+m} \phi(\boldsymbol{r})
\end{array}\right) .
$$

The expression from Eq. (3) is then obtained by setting $\phi(\boldsymbol{r})=e^{i\left(p_{x} x+p_{z} z\right)} I(y) \boldsymbol{s}$, where $\boldsymbol{s}$ is a 2-dimensional spinor and expanding $(\varepsilon-V(\boldsymbol{r})+m)^{-1} \simeq(\varepsilon+m)^{-1}\left(1+\frac{V(\boldsymbol{r})}{\varepsilon+m}\right)$ and keeping this correction term with the potential only on the $\sigma_{x} p_{x}$ term, as here this correction yields the leading order in $\xi / \gamma$ on $\sigma_{x}$.

\section{APPENDIX B}

The electron state can be written as (putting back in the volume factor)

$\psi_{p, s}(x)=\frac{1}{\sqrt{2 \varepsilon V}} e^{-i \varepsilon_{n} t} e^{i\left(p_{x} x+k_{B} y+p_{z} z\right)} \sum_{j} c_{j} \boldsymbol{S}_{j} e^{i j k_{0} y}$,

where $S_{j}$ is a bispinor defined as

$$
\boldsymbol{S}_{j}=\sqrt{\varepsilon+m}\left(\begin{array}{c}
\boldsymbol{s} \\
\frac{\sigma \cdot \boldsymbol{p}_{j}}{\varepsilon+m} \boldsymbol{s}
\end{array}\right),
$$


where $\boldsymbol{p}_{j}=\left(p_{x}+E_{n}-\frac{\left(j k_{0}+k_{B}\right)^{2}}{2 \varepsilon}, j k_{0}+k_{B}, p_{z}\right)$ and then

$$
\begin{aligned}
\int \psi_{p^{\prime}}^{\dagger} \psi_{p} d V= & \frac{1}{2 V \sqrt{\varepsilon^{\prime} \varepsilon}}(2 \pi)^{3} \delta\left(p_{x}-p_{x}^{\prime}\right) \delta\left(p_{z}-p_{z}^{\prime}\right) \\
& \times \sum_{j, j^{\prime}} c_{j}(p) c_{j^{\prime}}^{*}\left(p^{\prime}\right) \boldsymbol{S}_{j^{\prime}}^{\prime \dagger} \boldsymbol{S}_{j} \delta \\
& \times\left(k_{B}-k_{B}^{\prime}+\left(j-j^{\prime}\right) k_{0}\right) .
\end{aligned}
$$

Explicitly, we have that $c_{j}=c_{j}\left(\varepsilon, k_{B}, n\right)$. Now, since both $k_{B}$ and $k_{B}^{\prime}$ obey that $0 \leq k_{B}<k_{0}$, we have that $-k_{0}<$ $k_{B}-k_{B}^{\prime}<k_{0}$ and therefore $k_{B}-k_{B}^{\prime}$ can never be an integer value of $k_{0}$ unless $k_{B}-k_{B}^{\prime}=0$, and therefore we can write

$$
\begin{aligned}
\delta\left(k_{B}-\right. & \left.k_{B}^{\prime}+\left(j-j^{\prime}\right) k_{0}\right)=\delta\left(k_{B}-k_{B}^{\prime}\right) \delta_{j, j^{\prime}} \\
\int \psi_{p^{\prime}}^{\dagger} \psi_{p} d V= & \frac{1}{2 V \sqrt{\varepsilon^{\prime} \varepsilon}}(2 \pi)^{3} \delta\left(p_{x}-p_{x}^{\prime}\right) \delta\left(p_{z}-p_{z}^{\prime}\right) \\
& \times \delta\left(k_{B}-k_{B}^{\prime}\right) \sum_{j} c_{j}\left(\varepsilon, k_{B}, n\right) \\
& \times c_{j}^{*}\left(\varepsilon, k_{B}, n^{\prime}\right) \boldsymbol{S}_{j}^{\prime} \boldsymbol{S}_{j} .
\end{aligned}
$$

However, the vector $\boldsymbol{c}$ is a normalized $(|\boldsymbol{c}|=1)$ eigenvector of a Hermitian matrix, and the vectors corresponding to $n$ and $n^{\prime}$ have different eigenvalues of this matrix and are therefore orthogonal, so

$$
\begin{aligned}
\int \psi_{p^{\prime}}^{\dagger} \psi_{p} d V= & \frac{1}{2 V \sqrt{\varepsilon^{\prime} \varepsilon}}(2 \pi)^{3} \delta\left(p_{x}-p_{x}^{\prime}\right) \delta\left(p_{z}-p_{z}^{\prime}\right) \\
& \times \delta\left(k_{B}-k_{B}^{\prime}\right) \delta_{n, n^{\prime}} \sum_{j}\left|c_{j}\right|^{2} \boldsymbol{S}_{j}^{\prime \dagger} \boldsymbol{S}_{j} .
\end{aligned}
$$

Now, consider

$$
\begin{aligned}
\boldsymbol{S}_{j}^{\dagger \dagger} \boldsymbol{S}_{j} & =(\varepsilon+m)\left(\boldsymbol{s}^{\prime \dagger} \boldsymbol{s}+\boldsymbol{s}^{\prime \dagger} \frac{\boldsymbol{\sigma} \cdot \boldsymbol{p}_{j}}{\varepsilon+m} \frac{\boldsymbol{\sigma} \cdot \boldsymbol{p}_{j}}{\varepsilon+m} \boldsymbol{s}\right) \\
& =\boldsymbol{s}^{\prime \dagger} \boldsymbol{s}\left[(\varepsilon+m)+\frac{\boldsymbol{p}_{j}^{2}}{\varepsilon+m}\right],
\end{aligned}
$$

and therefore

$$
\sum_{j}\left|c_{j}\right|^{2} S_{j}^{\prime \dagger} S_{j} \simeq 2 \varepsilon \delta_{s^{\prime}, s}
$$

There, $\simeq$ refers only to the normalization. The states are exactly orthogonal, but in the normalization, we neglect corrections which are suppressed by at least $\xi / \gamma$ compared to leading order. So, finally,

$$
\begin{aligned}
\int \psi_{p^{\prime}}^{\dagger} \psi_{p} d V= & \frac{(2 \pi)^{3}}{V} \delta\left(p_{x}-p_{x}^{\prime}\right) \delta\left(p_{z}-p_{z}^{\prime}\right) \\
& \times \delta\left(k_{B}-k_{B}^{\prime}\right) \delta_{n, n^{\prime}} \delta_{s^{\prime}, s} .
\end{aligned}
$$

\section{APPENDIX C}

Inserting our wave functions into the expression from the paper, only the $y$ component is nontrivial:

$$
\begin{aligned}
\left\langle p_{y}\right\rangle & =\frac{1}{2 \varepsilon L} \int\left(e^{-i k_{B} y} \sum_{l} c_{l}^{*} \boldsymbol{S}_{l}^{\dagger} e^{-i l k_{0} y}\right)\left(-i \frac{d}{d y}\right)\left(e^{i k_{B} y} \sum_{j} c_{j} \boldsymbol{S}_{j} e^{i j k_{0} y}\right) d y \\
& =\frac{1}{2 \varepsilon L} \int\left(\sum_{l} c_{l}^{*} \boldsymbol{S}_{l}^{\dagger} e^{-i\left(l k_{0}+k_{B}\right) y}\right)\left(-i \frac{d}{d y}\right)\left(\sum_{j} c_{j} \boldsymbol{S}_{j} e^{i\left(j k_{0}+k_{B}\right) y}\right) d y \\
& =\frac{1}{2 \varepsilon L} \int\left(\sum_{l} c_{l}^{*} \boldsymbol{S}_{l}^{\dagger} e^{-i\left(l k_{0}+k_{B}\right) y}\right)\left(\sum_{j}\left(j k_{0}+k_{B}\right) c_{j} \boldsymbol{S}_{j} e^{i\left(j k_{0}+k_{B}\right) y}\right) d y \\
& =\frac{1}{2 \varepsilon L} \int\left(\sum_{j, l}\left(j k_{0}+k_{B}\right) c_{j} c_{l}^{*} \boldsymbol{S}_{l}^{\dagger} \boldsymbol{S}_{j} e^{i(j-l) k_{0} y}\right) d y .
\end{aligned}
$$

Now, due to periodicity of the integrand, we have that

$$
\frac{1}{L} \int_{-L / 2}^{L / 2} e^{i(j-l) k_{0} y} d y=\frac{1}{d_{p}} \int_{-d_{p} / 2}^{d_{p} / 2} e^{i(j-l) k_{0} y} d y=\operatorname{sinc}((j-l) \pi)=\delta_{j, l}
$$

and so

$$
\left\langle p_{y}\right\rangle=\frac{1}{2 \varepsilon}\left(\sum_{j}\left|c_{j}\right|^{2}\left(j k_{0}+k_{B}\right) \boldsymbol{S}_{j}^{\dagger} \boldsymbol{S}_{j}\right)=\frac{1}{2 \varepsilon}\left(\sum_{j}\left|c_{j}\right|^{2}\left(j k_{0}+k_{B}\right)\left((\varepsilon+m)+\frac{\boldsymbol{p}_{j}^{2}}{\varepsilon+m}\right)\right),
$$


and setting $p_{z}=0$,

$\boldsymbol{p}_{j}^{2}=\left(p_{x}+E_{n}-\frac{\left(j k_{0}+k_{B}\right)^{2}}{2 \varepsilon}\right)^{2}+\left(j k_{0}+k_{B}\right)^{2} \simeq p_{x}^{2}$,

and therefore

$$
\frac{1}{2 \varepsilon}\left((\varepsilon+m)+\frac{\boldsymbol{p}_{j}^{2}}{\varepsilon+m}\right) \simeq 1
$$

And so within our level of approximation, we have that

$$
\left\langle p_{y}\right\rangle \simeq \sum_{j}\left|c_{j}\right|^{2}\left(j k_{0}+k_{B}\right) .
$$

\section{APPENDIX D}

Starting from

$$
\begin{aligned}
S_{f i}^{(1)}= & i e \sqrt{\frac{4 \pi}{2 \omega}} \frac{1}{2 \sqrt{\varepsilon_{f} \varepsilon_{i}}}(2 \pi)^{3} \delta\left(p_{x, i}-k_{x}-p_{x, f}\right) \\
& \times \delta\left(p_{z, i}-k_{z}-p_{z, f}\right) \delta\left(\varepsilon_{f}+\omega-\varepsilon_{i}\right) \\
& \times \int_{-\infty}^{\infty} \bar{U}_{f}(y) \phi^{*} e^{i\left(k_{B, i}-k_{y}-K_{B, f}\right) y} U_{i}(y) d y,
\end{aligned}
$$

we insert the wave functions in terms of the plane wave expansion to obtain

$$
\begin{aligned}
S_{f i}^{(1)}= & i e \sqrt{\frac{4 \pi}{2 \omega}} \frac{1}{2 \sqrt{\varepsilon_{f} \varepsilon_{i}}}(2 \pi)^{3} \delta\left(p_{x, i}-k_{x}-p_{x, f}\right) \\
& \times \delta\left(p_{z, i}-k_{z}-p_{z, f}\right) \delta\left(\varepsilon_{f}+\omega-\varepsilon_{i}\right) \\
& \times \sum_{j, l} \int_{-\infty}^{\infty} c_{l, f}^{*} c_{j, i} \overline{\boldsymbol{S}}_{l, f} \phi^{*} \boldsymbol{S}_{j, i} e^{i\left(k_{B, i}-k_{y}-k_{B, f}\right) y} e^{i(j-l) k_{0} y} d y .
\end{aligned}
$$

The quantity $F=\int_{-\infty}^{\infty} e^{i\left(k_{B, i}-k_{y}-k_{B, f}\right) y} e^{i(j-l) k_{0} y} d y$ can be rewritten by exploiting that $e^{i(j-l) k_{0} y}$ is periodic, and so we see that

$$
F=\sum_{n=-\infty}^{\infty} \int_{-\frac{d_{p}}{2}+n d_{p}}^{\frac{d_{p}}{2}+n d_{p}} e^{i\left(k_{B, i}-k_{y}-k_{B, f}\right) y} e^{i(j-l) k_{0} y} d y
$$

and change the variable such that $y^{\prime}=y-n d_{p}$,

$$
\begin{aligned}
F= & \sum_{n=-\infty}^{\infty} \int_{-\frac{d_{p}}{2}}^{\frac{d_{p}}{2}} e^{i\left(k_{B, i}-k_{y}-k_{B, f}\right)\left(y^{\prime}+n d_{p}\right)} e^{i(j-l) k_{0} y^{\prime}} d y^{\prime} \\
= & \sum_{n=-\infty}^{\infty} e^{i n d_{p}\left(k_{B, i}-k_{y}-k_{B, f}\right)} \\
& \times \int_{-\frac{d_{p}}{2}}^{\frac{d_{p}}{2}} e^{i\left(k_{B, i}-k_{y}-k_{B, f}+(j-l) k_{0}\right) y^{\prime}} d y^{\prime} .
\end{aligned}
$$

Now, the sum $\sum_{n=-\infty}^{\infty} e^{i n d_{p}\left(k_{B, i}-k_{y}-k_{B, f}\right)}$ can be recognized as the Dirichlet kernel, which can be replaced with the Dirac comb $\sum_{n=-\infty}^{\infty} 2 \pi \delta\left(\left[k_{B, i}-k_{y}-k_{B, f}\right] d_{p}-2 \pi n\right)$. Only the delta function which has $k_{B, f}$ in the first Brillouin zone will contribute, due to the fact that integration limit on $k_{B, f}$ is from 0 to $k_{0}$, and therefore we must set $n=n_{B}$ such that $0 \leq k_{B, f}<k_{0}$. Therefore, we may use that

$$
\begin{aligned}
F= & 2 \pi \delta\left(\left[k_{B, i}-k_{y}-k_{B, f}\right]-n_{B} k_{0}\right) \\
& \times \operatorname{sinc}\left(\left[\pi n_{B}+(j-l) \pi\right]\right) .
\end{aligned}
$$

This simplifies the summation over $l$ as the sinc function means that only the term obeying $n_{B}+(j-l)=0$ contributes. Therefore, the S-matrix element becomes

$$
\begin{aligned}
S_{f i}^{(1)}= & i e \sqrt{\frac{4 \pi}{2 \omega}} \frac{1}{2 \sqrt{\varepsilon_{f} \varepsilon_{i}}}(2 \pi)^{4} \delta\left(p_{x, i}-k_{x}-p_{x, f}\right) \delta \\
& \times\left(p_{z, i}-k_{z}-p_{z, f}\right) \delta\left(\varepsilon_{f}+\omega-\varepsilon_{i}\right) \\
& \times \delta\left(\left[k_{B, i}-k_{y}-k_{B, f}\right]-n_{B} k_{0}\right) \\
& \times \sum_{j} c_{n_{B}+j, f}^{*} c_{j, i} \overline{\boldsymbol{S}}_{n_{B}+j, f} \phi^{\phi^{*} \boldsymbol{S}_{j, i} .}
\end{aligned}
$$

Now, a useful expression for the quantity $\overline{\boldsymbol{S}}_{n_{B}+j, f} \phi^{*} \boldsymbol{S}_{j, i}$ may be derived. We will momentarily suppress the $j$ index, and so

$$
\begin{aligned}
& -\overline{\boldsymbol{S}}_{f} \phi^{*} \boldsymbol{S}_{i}=\sqrt{\left(\varepsilon_{f}+m\right)\left(\varepsilon_{i}+m\right)}\left(\begin{array}{ll}
\boldsymbol{s}_{f}^{\dagger} & \boldsymbol{s}_{f}^{\dagger} \frac{\boldsymbol{\sigma} \cdot \boldsymbol{p}_{f}}{\varepsilon_{f}+m}
\end{array}\right)\left(\boldsymbol{\alpha} \cdot \boldsymbol{\epsilon}^{*}\right)\left(\begin{array}{c}
\boldsymbol{s}_{i} \\
\frac{\boldsymbol{\sigma} \cdot \boldsymbol{p}_{i}}{\bar{\varepsilon}_{i}+m} \boldsymbol{s}_{i}
\end{array}\right) \\
& =\sqrt{\left(\varepsilon_{f}+m\right)\left(\varepsilon_{i}+m\right)}\left(\begin{array}{ll}
\boldsymbol{s}_{f}^{\dagger} & \boldsymbol{s}_{f}^{\dagger} \frac{\boldsymbol{\sigma} \cdot \boldsymbol{p}_{f}}{\varepsilon_{f}+m}
\end{array}\right)\left(\begin{array}{cc}
0 & \boldsymbol{\sigma} \cdot \boldsymbol{\epsilon}^{*} \\
\boldsymbol{\sigma} \cdot \boldsymbol{\epsilon}^{*} & 0
\end{array}\right)\left(\begin{array}{c}
\boldsymbol{s}_{i} \\
\frac{\boldsymbol{\sigma} \cdot p_{i}}{\varepsilon_{i}+m} \boldsymbol{s}_{i}
\end{array}\right) \\
& =\sqrt{\left(\varepsilon_{f}+m\right)\left(\varepsilon_{i}+m\right)}\left(\begin{array}{ll}
\boldsymbol{s}_{f}^{\dagger} & \boldsymbol{s}_{f}^{\dagger} \frac{\boldsymbol{\sigma} \cdot \boldsymbol{p}_{f}}{\varepsilon_{f}+m}
\end{array}\right)\left(\begin{array}{c}
\boldsymbol{\sigma} \cdot \boldsymbol{\epsilon}^{*} \frac{\boldsymbol{\sigma} \cdot \boldsymbol{p}_{i}}{\varepsilon_{i}+m} \boldsymbol{s}_{i} \\
\boldsymbol{\sigma} \cdot \boldsymbol{\epsilon}^{*} \boldsymbol{s}_{i}
\end{array}\right) \\
& =\sqrt{\left(\varepsilon_{f}+m\right)\left(\varepsilon_{i}+m\right)} \boldsymbol{s}_{f}^{\dagger}\left(\boldsymbol{\sigma} \cdot \boldsymbol{\epsilon}^{*} \frac{\boldsymbol{\sigma} \cdot \boldsymbol{p}_{i}}{\varepsilon_{i}+m}+\frac{\boldsymbol{\sigma} \cdot \boldsymbol{p}_{f}}{\varepsilon_{f}+m} \boldsymbol{\sigma} \cdot \boldsymbol{\epsilon}^{*}\right) \boldsymbol{s}_{i} \\
& =\sqrt{\left(\varepsilon_{f}+m\right)\left(\varepsilon_{i}+m\right)} \boldsymbol{s}_{f}^{\dagger}\left(\frac{1}{\varepsilon_{i}+m}\left[\boldsymbol{\epsilon}^{*} \cdot \boldsymbol{p}_{i}+i \boldsymbol{\sigma} \cdot\left(\boldsymbol{\epsilon}^{*} \times \boldsymbol{p}_{i}\right)\right]+\frac{1}{\varepsilon_{f}+m}\left[\boldsymbol{p}_{f} \cdot \boldsymbol{\epsilon}^{*}+i \boldsymbol{\sigma} \cdot\left(\boldsymbol{p}_{f} \times \boldsymbol{\epsilon}^{*}\right)\right]\right) \boldsymbol{s}_{i} \\
& =\sqrt{\left(\varepsilon_{f}+m\right)\left(\varepsilon_{i}+m\right)} \boldsymbol{s}_{f}^{\dagger}\left[\boldsymbol{\epsilon}^{*} \cdot \boldsymbol{A}+i \boldsymbol{\sigma} \cdot \boldsymbol{B}\right] \boldsymbol{s}_{i},
\end{aligned}
$$


where we have defined

$$
\begin{gathered}
\boldsymbol{A}=\frac{1}{\varepsilon_{i}+m} \boldsymbol{p}_{i}+\frac{1}{\varepsilon_{f}+m} \boldsymbol{p}_{f}, \\
\boldsymbol{B}=\boldsymbol{\epsilon}^{*} \times\left(\frac{\boldsymbol{p}_{i}}{\varepsilon_{i}+m}-\frac{\boldsymbol{p}_{f}}{\varepsilon_{f}+m}\right) .
\end{gathered}
$$

[1] J. Lindhard, Influence of crystal lattice on motion of energetic charged particles, K. Dan. Vidensk. Selsk. Mat. Fys. Medd. 34, 1 (1965).

[2] J. Bak, J. A. Ellison, B. Marsh, F. E. Meyer, O. Pedersen, J. B. B. Petersen, E. Uggerhøj, K. Østergaard, S. P. Møller, A. H. Sørensen, and M. Suffert, Channeling radiation from 2-55 GeV/c electrons and positrons: (I). Planar case, Nucl. Phys. B254, 491 (1985).

[3] J. F. Bak, J. A. Ellison, B. Marsh, F. E. Meyer, O. Pedersen, J. B. B. Petersen, E. Uggerhøj, S. P. Møller, H. Sørensen, and M. Suffert, Channeling radiation from 2 to $20 \mathrm{GeV} / \mathrm{c}$ electrons and positrons (II): Axial case, Nucl. Phys. B302, 525 (1988).

[4] R. L. Swent, R. H. Pantell, M. J. Alguard, B. L. Berman, S.D. Bloom, and S. Datz, Observation of Channeling Radiation from Relativistic Electrons, Phys. Rev. Lett. 43, 1723 (1979).

[5] J. U. Andersen, K. R. Eriksen, and E. Laegsgaard, PlanarChanneling Radiation and Coherent Bremsstrahlung for MeV Electrons, Phys. Scr. 24, 588 (1981).

[6] J. U. Andersen, E. Bonderup, E. Laegsgaard, B. B. Marsh, and A. H. Sørensen, Axial channeling radiation from $\mathrm{MeV}$ electrons, Nucl. Instrum. Methods Phys. Res. 194, 209 (1982).

[7] R. K. Klein, J. O. Kephart, R. H. Pantell, H. Park, B. L. Berman, R. L. Swent, S. Datz, and R. W. Fearick, Electron channeling radiation from diamond, Phys. Rev. B 31, 68 (1985).

[8] M. J. Alguard, R. L. Swent, R. H. Pantell, B. L. Berman, S. D. Bloom, and S. Datz, Observation of Radiation from Channeled Positrons, Phys. Rev. Lett. 42, 1148 (1979).

[9] K. K. Andersen, J. Esberg, H. Knudsen, H. D. Thomsen, U. I. Uggerhøj, P. Sona, A. Mangiarotti, T. J. Ketel, A. Dizdar, and S. Ballestrero (CERN NA63 Collaboration), Experimental investigations of synchrotron radiation at the onset of the quantum regime, Phys. Rev. D 86, 072001 (2012).

[10] U. I. Uggerh $ø$, The interaction of relativistic particles with strong crystalline fields, Rev. Mod. Phys. 77, 1131 (2005).

[11] T. N. Wistisen, A. Di Piazza, H. V. Knudsen, and U. I. Uggerhøj, Experimental evidence of quantum radiation reaction in aligned crystals, Nat. Commun. 9, 795 (2018).

[12] T. N. Wistisen, K. K. Andersen, S. Yilmaz, R. Mikkelsen, J. L. Hansen, U. I. Uggerhøj, W. Lauth, and H. Backe, Experimental Realization of a New Type of Crystalline Undulator, Phys. Rev. Lett. 112, 254801 (2014).
[13] T. N. Wistisen, U. I. Uggerhøj, J. L. Hansen, W. Lauth, and P. Klag, Radiation collimation in a thick crystalline undulator, Eur. Phys. J. D 71, 124 (2017).

[14] M. A. Kumakhov, On the theory of electromagnetic radiation of charged particles in a crystal, Phys. Lett. 57A, 17 (1976).

[15] M. A. Kumakhov, Theory of radiation of charged particles channeled in a crystal, Phys. Status Solidi B 84, 41 (1977).

[16] A. W. Sáenz, H. Überall, and A. Nagl, Calculation of electron channeling radiation with a realistic potential, Nucl. Phys. A372, 90 (1981).

[17] V. V. Beloshitskii and A. Kh. Khokonov, A study of the hard part of the electromagnetic radiation spectrum of positrons passing through single crystals, Sov. Phys. Tech. Phys. 32, 1182 (1987).

[18] C. Bula, K. T. McDonald, E. J. Prebys, C. Bamber, S. Boege, T. Kotseroglou, A. C. Melissinos, D. D. Meyerhofer, W. Ragg, D. L. Burke et al., Observation of Nonlinear Effects in Compton Scattering, Phys. Rev. Lett. 76, 3116 (1996).

[19] J.M. Cole et al., Experimental Evidence of Radiation Reaction in the Collision of a High-Intensity Laser Pulse with a Laser-Wakefield Accelerated Electron Beam, Phys. Rev. X 8, 011020 (2018).

[20] K. Poder et al., Experimental Signatures of the Quantum Nature of Radiation Reaction in the Field of an Ultraintense Laser, Phys. Rev. X 8, 031004 (2018).

[21] V. I. Ritus, Quantum effects of the interaction of elementary particles with an intense electromagnetic field, J. Sov. Laser Res. 6, 497 (1985).

[22] M. Boca and V. Florescu, Nonlinear Compton scattering with a laser pulse, Phys. Rev. A 80, 053403 (2009).

[23] V. Dinu and G. Torgrimsson, Single, double and higherorder nonlinear Compton scattering, Phys. Rev. D 99, 096018 (2019).

[24] D. Seipt and B. Kämpfer, Nonlinear Compton scattering of ultrashort intense laser pulses, Phys. Rev. A 83, 022101 (2011).

[25] F. Mackenroth and A. Di Piazza, Nonlinear Compton scattering in ultrashort laser pulses, Phys. Rev. A 83, 032106 (2011).

[26] F. Mackenroth and A. Di Piazza, Nonlinear Double Compton Scattering in the Ultrarelativistic Quantum Regime, Phys. Rev. Lett. 110, 070402 (2013). 
[27] B. King, Double Compton scattering in a constant crossed field, Phys. Rev. A 91, 033415 (2015).

[28] P. Lervig, J. Lindhard, and V. Nielsen, Quantal treatment of directional effects for energetic charged particles in crystal lattices, Nucl. Phys. A96, 481 (1967).

[29] J. U. Andersen and W. M. Augustyniak, Channeling of electrons and positrons, K. Dan. Vidensk. Selsk. Mat. Fys. Medd. 89, 1 (1977).

[30] V. N. Baier and V. M. Katkov, Processes involved in the motion of high energy particles in a magnetic field, J. Exp. Theor. Phys. 26, 854 (1968).

[31] A. V. Korol, A. V. Solov'yov, and W. Greiner, Channeling and Radiation in Periodically Bent Crystals (Springer, Berlin, Heidelberg, 2013).

[32] L. Bandiera, E. Bagli, V. Guidi, and V. V. Tikhomirov, RADCHARM++: A C++ routine to compute the electromagnetic radiation generated by relativistic charged particles in crystals and complex structures, Nucl. Instrum. Methods Phys. Res., Sect. B 355, 44 (2015).

[33] V. Guidi, L. Bandiera, and V. V. Tikhomirov, Radiation generated by single and multiple volume reflection of ultrarelativistic electrons and positrons in bent crystals, Phys. Rev. A 86, 042903 (2012).

[34] M. A. Kumakhov and R. Wedell, Theory of radiation of relativistic channelled particles, Phys. Status Solidi B 84, 581 (1977).

[35] N. K. Zhevago, Emission of gamma rays by channeled particles, J. Exp. Theor. Phys. 48, 701 (1978).

[36] V. V. Beloshitskii and M. A. Kumakhov, Quantum theory of spontaneous and induced radiation of channeled electrons and positrons, J. Exp. Theor. Phys. 47, 652 (1978).

[37] V. A. Bazylev, V. I. Glebov, and N. K. Zhevago, Spectral distribution of the radiation in planar and axial channeling of ultrarelativistic electrons, J. Exp. Theor. Phys. 51, 31 (1980).

[38] J. C. Kimball and N. Cue, Quantum electrodynamics and channeling in crystals, Phys. Rep. 125, 69 (1985).

[39] S. N. Shulga, N. F. Shul'ga, S. Barsuk, I. Chaikovska, and R. Chehab, On classical and quantum effects at scattering of ultrarelativistic electrons in ultrathin crystal, Nucl. Instrum. Methods Phys. Res., Sect. B 402, 16 (2017); Proceedings of the 7th International Conference Channeling 2016: Charged and Neutral Particles Channeling Phenomena (Elsevier Science, Amsterdam, Netherlands, 2017).

[40] A. V. Kozlov, N. F. Shul'ga, and V. A. Cherkaskiy, Spectral method in quantum theory of channeling phenomena of fast charged particles in crystals, Phys. Lett. A 374, 4690 (2010).

[41] N. F. Shul'ga, V. V. Syshchenko, A. I. Tarnovsky, and A. Yu. Isupov, Structure of the channeling electrons wave functions under dynamical chaos conditions, Nucl. Instrum. Methods Phys. Res., Sect. B 370, 1 (2016).

[42] A. Di Piazza, T. N. Wistisen, and U. I. Uggerhøj, Investigation of classical radiation reaction with aligned crystals, Phys. Lett. B 765, 1 (2017).

[43] S. V. Abdrashitov, O. V. Bogdanov, P. O. Kazinski, and T. A. Tukhfatullin, Orbital angular momentum of channeling radiation from relativistic electrons in thin Si crystal, Phys. Lett. A 382, 3141 (2018).

[44] L. Bandiera, E. Bagli, G. Germogli, V. Guidi, A. Mazzolari, H. Backe, W. Lauth, A. Berra, D. Lietti, M. Prest, D. De
Salvador, E. Vallazza, and V. Tikhomirov, Investigation of the Electromagnetic Radiation Emitted by Sub-Gev Electrons in a Bent Crystal, Phys. Rev. Lett. 115, 025504 (2015).

[45] T. N. Wistisen, R.E. Mikkelsen, U. I. Uggerhøj, U. Wienands, T. W. Markiewicz, S. Gessner, M. J. Hogan, R. J. Noble, R. Holtzapple, S. Tucker, V. Guidi, A. Mazzolari, E. Bagli, L. Bandiera, and A. Sytov (SLAC E-212 Collaboration), Observation of Quasichanneling Oscillations, Phys. Rev. Lett. 119, 024801 (2017).

[46] A. V. Korol, V. G. Bezchastnov, G. B. Sushko, and A. V. Solov'yov, Simulation of channeling and radiation of $855 \mathrm{MeV}$ electrons and positrons in a small-amplitude short-period bent crystal, Nucl. Instrum. Methods Phys. Res., Sect. B 387, 41 (2016).

[47] L. Bandiera, E. Bagli, V. Guidi, A. Mazzolari, A. Berra, D. Lietti, M. Prest, E. Vallazza, D. De Salvador, and V. Tikhomirov, Broad and Intense Radiation Accompanying Multiple Volume Reflection of Ultrarelativistic Electrons in a Bent Crystal, Phys. Rev. Lett. 111, 255502 (2013).

[48] H. Backe, D. Krambrich, W. Lauth, K. K. Andersen, J. Lundsgaard Hansen, and Ulrik I. Uggerhøj, Radiation emission at channeling of electrons in a strained layer $S i_{1-x} G e_{x}$ undulator crystal, Nucl. Instrum. Methods Phys. Res., Sect. B 309, 37 (2013); Proceedings of the 5th International Conference "Channeling 2012", Charged \& Neutral Particles Channeling Phenomena, 2012, Alghero (Sardinia), Italy (Elsevier Science, Amsterdam, Netherlands, 2013).

[49] U. Wienands, T. W. Markiewicz, J. Nelson, R. J. Noble, J. L. Turner, U. I. Uggerhøj, T. N. Wistisen, E. Bagli, L. Bandiera, G. Germogli, V. Guidi, A. Mazzolari, R. Holtzapple, and M. Miller, Observation of Deflection of a Beam of Multi-Gev Electrons by a Thin Crystal, Phys. Rev. Lett. 114, 074801 (2015).

[50] T. N. Wistisen, U. I. Uggerhøj, U. Wienands, T. W. Markiewicz, R. J. Noble, B. C. Benson, T. Smith, E. Bagli, L. Bandiera, G. Germogli, V. Guidi, A. Mazzolari, R. Holtzapple, and S. Tucker, Channeling, volume reflection, and volume capture study of electrons in a bent silicon crystal, Phys. Rev. Accel. Beams 19, 071001 (2016).

[51] Andriy Kostyuk, Crystalline Undulator with a Small Amplitude and a Short Period, Phys. Rev. Lett. 110, 115503 (2013).

[52] A. Kh. Khokonov, M. Kh. Khokonov, and A. A. Kizdermishov, Possibility of generating high-energy photons by ultrarelativistic electrons in the field of a terawatt laser and in crystals, Tech. Phys. 47, 1413 (2002).

[53] M. Kh. Khokonov, On the quantum interpretation of the classical schott term in the theory of radiation damping, Phys. Lett. B 791, 281 (2019).

[54] T. N. Wistisen and A. Di Piazza, Impact of the quantized transverse motion on radiation emission in a Dirac harmonic oscillator, Phys. Rev. A 98, 022131 (2018).

[55] V. N. Baier, V. M. Katkov, and V. M. Strakhovenko, Electromagnetic Processes at High Energies in Oriented Single Crystals (World Scientific, Singapore, 1998).

[56] P. A. Doyle and P. S. Turner, Relativistic Hartree-Fock X-ray and electron scattering factors, Acta Crystallogr. A 24, 390 (1968). 
[57] A. L. Avakian, N. K. Zhevago, and Shi Yan, Emission of electrons and positrons in the axial semichanneling, J. Exp. Theor. Phys. 82, 573 (1982).

[58] S. P. Møller, High-energy channeling - applications in beam bending and extraction, Nucl. Instrum. Methods Phys. Res., Sect. A 361, 403 (1995).

[59] N. Ashcroft and N. Mermin, Solid State Physics, International Edition (Brooks/Cole, Belmont, CA, 1976).

[60] J. D. Jackson, Classical Electrodynamics (Wiley, Hoboken, NJ, 1999).
[61] T. N. Wistisen, Interference effect in nonlinear Compton scattering, Phys. Rev. D 90, 125008 (2014).

[62] T. N. Wistisen, Quantum synchrotron radiation in the case of a field with finite extension, Phys. Rev. D 92, 045045 (2015).

[63] E. Raicher, S. Eliezer, C. H. Keitel, and K. Z. Hatsagortsyan, Semi-classical limitations for photon emission in strong external fields, arXiv:1810.07523.

[64] M. Tanabashi et al. (Particle Data Group), Review of particle physics, Phys. Rev. D 98, 030001 (2018). 